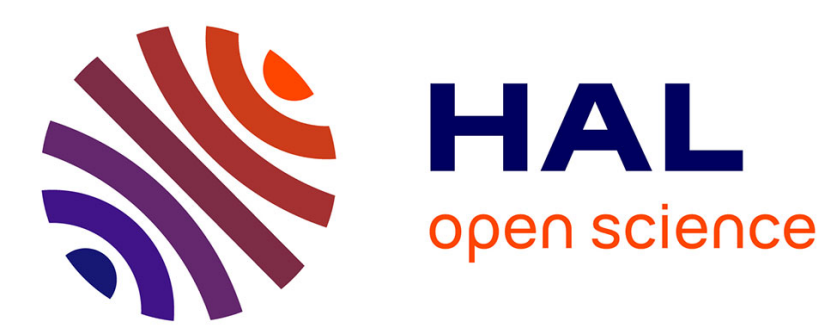

\title{
Note sur l'emploi et le choix des lunettes destinées à corriger les mauvaises vues
}

Emeric Dubois

\section{To cite this version:}

Emeric Dubois. Note sur l'emploi et le choix des lunettes destinées à corriger les mauvaises vues. J. Phys. Theor. Appl., 1877, 6 (1), pp.28-29. 10.1051/jphystap:01877006002801 . jpa-00237311

\section{HAL Id: jpa-00237311 https://hal.science/jpa-00237311}

Submitted on 1 Jan 1877

HAL is a multi-disciplinary open access archive for the deposit and dissemination of scientific research documents, whether they are published or not. The documents may come from teaching and research institutions in France or abroad, or from public or private research centers.
L'archive ouverte pluridisciplinaire HAL, est destinée au dépôt et à la diffusion de documents scientifiques de niveau recherche, publiés ou non, émanant des établissements d'enseignement et de recherche français ou étrangers, des laboratoires publics ou privés. 


\section{NOTE SUR L'EMPLOI ET LE GHOIX DES LUNETTES DESTINÉES A CORRIGER LES MAOVAISES VOES;}

Par M. E. DUBOIS, Professeur au lycse d'Amiens.

On sait que le but des lunettes est de permettre aux myopes et aux presbytes de voir nettement les objets, en les plaçant à la distance $\mathrm{D}$ de la vision distincte d'un œil normal. Il s'agit donc, en plaçant un objet à une distance $\mathrm{D}$ du centre optique de l'œil, d'en observer une image virtuelle à une distance $\mathrm{D}^{\prime}<\mathrm{D}$ pourles myopes, et à une distance $D_{1}^{\prime}>D$ pour les presbytes. La solution du problème est donnée dans tous les Traités de Physique. Une lentille divergente corrige la vue des myopes et une lentille convergente celle des presbytes. Mais, dans le calcul de la distance focale qui convient à une vue donnée, on néglige toujours de tenir compte de la distance de la lentille employée à l'œil, distance qui n'est pas sans importance.

Cherchons la distance focale, $f$ ou $f_{1}$, de la lentille, d'épaisseur négligeable, convenant à une vue donnée, et appelons $a$ la distance du centre optique de cette lentille au centre optique, ou mieux au premier point nodal de l'œil. On aura, en appliquant la formule des lentilles et mettant les signes en évidence, pour les myopes,

$$
-\frac{\mathrm{I}}{\mathrm{D}-a}+\frac{\mathrm{I}}{\mathbf{D}^{\prime}-a}=\frac{\mathrm{I}}{f}
$$

d'où

$$
f=\frac{(\mathrm{D}-a)\left(\mathrm{I}^{\prime}-a\right)}{\mathrm{I}-\mathrm{I}^{\prime}}
$$

pour les presbytes,

$$
\frac{\mathrm{I}}{\mathrm{D}-a}-\frac{\mathrm{I}}{\mathrm{D}_{1}^{\prime}-a}=\frac{\mathrm{I}}{f_{1}}
$$



d'où

$$
f_{1}=\frac{(\mathrm{D}-a)\left(\mathrm{D}_{1}^{\prime}-a\right)}{\mathrm{D}_{1}^{\prime}-\mathrm{D}} .
$$

Ces formules montrent un fait intéressant : c'est que $f$ et $f_{1}$ décroissent lorsque $a$ croit; on a, en effet,

$$
\frac{d f}{d a}=\frac{2 a-\left(\mathbf{D}+\mathbf{D}^{\prime}\right)}{\mathbf{D}-\mathbf{D}^{\prime}}, \quad \frac{d f_{1}}{d a}=\frac{2 a-\left(\mathbf{D}+\mathbf{D}_{1}^{\prime}\right)}{\mathbf{D}_{1}^{\prime}-\mathbf{D}} .
$$

Ces dérivées sont toujours négatives pour

$$
a<\frac{\mathbf{D}+\mathbf{D}^{\prime}}{2},
$$

condition toujours réalisée dans l'emploi des lunettes; donc, pour une vue donnée, il faudra employer une lentille de distance focale d'autant plus faible qu'on mettra le verre plus loin de l'œil. De là le conseil souvent donné de placer les verres aussi près que possible de l'œil, pour éviter l'emploi de verres trop forts; de là aussi l'habitude des médecins militaires qui appliquent les lunettes contre les yeux des conscrits atteints de myopie.

Les formules (I) et (2) montrent encore que la lentille doit être à foyer d'autant plus court que l'on est plus myope ou plus presbyte.

On a, en effet, pour les myopes,

$$
\frac{d f}{d \mathbf{D}^{\prime}}=\frac{(\mathbf{D}-a)^{2}}{\left(\mathbf{D}-\mathbf{D}^{\prime}\right)^{2}}
$$

quantité essentiellement positive : donc $f$ décroit quand $\mathrm{D}^{\prime}$ décroît; et pour les presbytes,

$$
\frac{d f_{1}}{d \mathbf{D}_{1}^{\prime}}=\frac{-(\mathbf{D}-a)^{2}}{\left(\mathbf{D}_{1}^{\prime}-\mathbf{D}\right)^{2}}
$$

quantité essentiellement négative : donc $f_{1}$ décroît lorsque $\mathrm{D}_{1}^{\prime}$ croît.

IH. HELMHOLTZ. - Bericht betreffend Versuche über die elektromagnetische Wirkung electrischer Convection, etc. (Rapport sur des expériences relatives à l'action électromagnétique de l'électricité transportée par la matière pondérable en mouvement, exécutées par M. Henry Rowiand, de Baltimore); Ann. de Pogg., t. CLVIII, p. $\bigwedge_{9} 7$, I 876 .

La question de savoir si une molécule matérielle chargée d'élec- 